\title{
Loading BMP-2 on nanostructured hydroxyapatite microspheres for rapid bone regeneration
}

This article was published in the following Dove Press journal:

International Journal of Nanomedicine

\author{
Panyu Zhou ${ }^{1, *}$ \\ Jianghong $\mathrm{Wu}^{1, *}$ \\ Yan Xia ${ }^{1, *}$ \\ Ye Yuan' \\ Hongyue Zhang' \\ Shuogui $\mathrm{Xu}$ \\ Kaili Lin ${ }^{2}$ \\ 'Department of Emergency, \\ Changhai Hospital, Second Military \\ Medical University, Shanghai \\ 200433, People's Republic of China; \\ 2Department of Oral \& Cranio- \\ Maxillofacial Surgery, Shanghai Ninth \\ People's Hospital, Shanghai Jiao \\ Tong University School of Medicine, \\ Shanghai 2000 II, People's Republic \\ of China \\ *These authors contributed equally \\ to this work
}

Introduction: Tissue engineering is a promising strategy for bone regeneration in repairing massive bone defects. The surface morphology of implanted materials plays a key role in bone healing; these materials incorporate osteoinductive factors to improve the efficiency of bone regeneration.

Materials and methods: In the current study, nanostructured hydroxyapatite (nHAp) microspheres were prepared via a hydrothermal transformation method using calcium silicate (CS) microspheres as precursors; the CS microspheres were obtained by a spray-drying method. The nHAp microspheres constructed by the nano-whiskers significantly improved the ability of the microspheres to adsorb the bioactive protein (BMP-2) and reduce its initial burst release. To evaluate the in vivo bone regeneration of microspheres, both conventional hydroxyapatite (HAp) and nHAp microspheres were either loaded with recombinant human bone morphogenetic protein-2 (rhBMP-2) or not loaded with the protein; these microspheres were implanted in rat femoral bone defects for 4 and 8 weeks.

Results and discussion: The results of our three-dimensional (3D) micro-computed tomography (CT) and histomorphometric observations showed that the combination of the nanostructured surface and rhBMP-2 obviously improved osteogenesis compared to conventional HAp microspheres loaded with rhBMP-2. Our results suggest that the nHAp microspheres with a nanostructured surface adsorb rhBMP-2 for rapid bone formation; they therefore show the potential to act as carriers in bone tissue regeneration.

Keywords: BMP-2, nanostructure, hydroxyapatite, hydrothermal transformation, bone regeneration

\section{Introduction}

Bone transplantation is one of the most popular allogeneic tissue transfusions, which is second only to blood transfusions in frequency. ${ }^{1}$ Bone transplantation is widely used in clinics, such as for the treatment of fractures, delayed healing, limb preservation after bone tumor resection, trauma, infectious bone defects, joint fusion, and joint repair. The autologous bone is particularly important in the treatment of fracture, bone nonunion, oral tooth planting, and mandibular hypoplasia..$^{2-4}$ Moreover, autologous bone grafts have good tissue compatibility and retain osteoblasts, cytokines, and other active substances present in bone which effectively promote osteogenesis. ${ }^{5}$ These grafts are the gold standard for bone defect repair, but their clinical applications are limited for many reasons. ${ }^{6-8}$ The first reason for their restricted application is that the autologous bone supply is extremely limited. The donor site is even more restricted in the elderly and children, and it is difficult to meet the needs of clinical practice. Second, autologous bone grafting inevitably destroys the normal tissue and structure of the donor site, which results in bleeding, infection, pain, postoperative scar hyperplasia, and other 
complications. Finally, additional surgical procedures are inevitable, and these can lead to prolonged surgical duration and anesthesia time, increasing the risk of the surgeries.

In contrast, there are many sources of xenogeneic bone grafts. They are easy to harvest, but they are limited because of religious/ethical concerns and immune rejection. ${ }^{9}$ It is possible to remove the organic components of xenogeneic bone via special physical and chemical processing techniques, which also eliminate its antigenicity. However, such processing will also inevitably destroy the biological activity of the xenogeneic bone, resulting in a loss of the osteoinductive activity, and ultimately reduce the mechanical strength and plasticity of the implants. Xenogeneic bone transplantation is further challenged by the fear of virus transmission, including spongiform encephalopathy. ${ }^{10}$

Thus, tissue engineering has considerable potential to improve the bone regeneration techniques that are currently employed in bone implant surgery. ${ }^{10}$ The scaffolds incorporated with endogenous osteoinductive growth factors or other signaling molecules are capable of promoting bone regeneration by serving as matrices for tissue formation. ${ }^{1-13}$ However, inappropriate biofactor delivery strategies and the high technical requirements of implants often lead to failures in surgery. ${ }^{14,15}$ The alternative implant materials with the ability of osteogenetic induction and easy to perform are therefore significantly important in scientific and clinical field. We addressed the problems mentioned earlier by developing simple and stable implants with enhanced osteoinductivity and improved implant feasibility.

Bone morphogenetic protein-2 (BMP-2), one of the important endogenous growth factors, can promote the proliferation of mesenchymal cells and induce the differentiation of mesenchymal cells into bone tissue. By enhancing or inhibiting the secretion of certain specific proteins in target cells, BMP-2 induces undifferentiated mesenchymal cells into bone formation centers where they differentiate into bone-related cells. BMP-2 also makes fibroblasts to differentiate into osteoblasts and myoblasts to differentiate into chondrocytes, which promotes matrix calcification. In addition, BMP-2 reverses the differentiation of bone marrow basal cells into bone cells, which can induce osteogenesis and hence bone formation with the high expression of alkaline phosphatase as well as BMP simultaneously. ${ }^{16-18}$ Some biomaterials were reported to successfully load and retain recombinant human bone morphogenetic protein-2 (rhBMP-2) using particular microstructures and then release it as needed, which indicated great biological potential. Scaffolds as bioactive ingredient delivery systems were also developed for treating bone defects and optimizing therapeutic efficacy. ${ }^{19,20}$ However, the initial burst release of the loaded growth factors from scaffolds significantly reduces their bioutility (particularly rhBMP-2) and therefore remains an obstacle to clinical use. Some studies have shown that if micro-/nano-sized pores are introduced into calcium phosphate bioceramics - or the sizes of the grains at the material surface are controlled at the micro/nano level - then these ceramics can effectively simulate the structure and size of the collagen fibers of the body's natural bone tissue. This stimulation can greatly improve the osteogenic activity of the materials and even endow the material with the ability to induce ectopic osteogenesis. ${ }^{21-23}$

Current synthetic bone materials, such as bioglass, calcium phosphate cement, polymethyl methacrylate, and composite materials, have been tentatively used as carriers for implanting active molecules or target proteins into the body. However, due to the limitation of the structure of the material itself, the target protein cannot be effectively delivered to the defect site, and their role in promoting bone repair is limited. This effect may be due to the introduction of the micro-/nano-sized pores and grains, large and highly ordered pores and uniform tunable channels, which vary in diameter from 5 to $30 \mathrm{~nm}$ that increase the specific surface area of the material so that it has a good ability to recruit bioactive substances. ${ }^{24-26}$ The structural modification of a bioceramic surface with micro/nanostructures can also significantly improve the ability of the material to adsorb bioactive proteins and promote cell adhesion, proliferation, and differentiation on the surface of the materials, which may ultimately promote osteogenesis. ${ }^{27,28}$ More importantly, our previous studies showed that the hydroxyapatite (HAp) bioceramics with micro/nanostructured surfaces could significantly promote cell attachment, proliferation, and osteogenic differentiation of osteoblast and bone marrow stromal cells in vitro and enhance bone regeneration in vivo. ${ }^{29,30}$

In this study, nanostructured HAp $\left(\mathrm{nHAp}, \mathrm{Ca}_{10}\left(\mathrm{PO}_{4}\right)_{6}(\mathrm{OH})_{2}\right)$ microspheres were prepared via a hydrothermal transformation method using calcium silicate $\left(\mathrm{CS}, \mathrm{CaSiO}_{3}\right)$ microspheres as precursors; these CS microspheres were obtained using a spray-drying method. The sphere-like structures can serve as injectable bone-filling implants, especially for defects with irregular and complex shapes and sizes. In addition, the spaces constructed by the spheres after implantation are vital for functional and effective bone regeneration, and they also allow for bone and vascular ingrowths. nHAp microspheres have the advantage of the localized and sustained release of therapeutically relevant factors, and so they are often used as carriers. ${ }^{31}$ Thus, rhBMP-2 was incorporated into the nHAp microspheres to effectively reduce its initial burst release and prolong its residence time at the site of bone injury. 
The performance of the rhBMP-2-loaded nHAp microspheres on the rapid bone regeneration was also investigated using a model.

\section{Materials and methods}

\section{Fabrication and characterization of $\mathrm{nHAp}$ microspheres}

The nHAp microspheres were prepared via a hydrothermal transformation of CS microspheres as precursors in a trisodium phosphate aqueous solution. The CS microspheres were fabricated via the spray-drying method that was used in our previous study. ${ }^{32}$ First, the CS powders were synthesized via the chemical precipitation method by the reaction of $\mathrm{Na}_{2} \mathrm{SiO}_{3} \cdot 9 \mathrm{H}_{2} \mathrm{O}$ and $\mathrm{Ca}\left(\mathrm{NO}_{3}\right)_{2} \cdot 4 \mathrm{H}_{2} \mathrm{O}$ aqueous solutions. ${ }^{33}$ The chemically precipitated CS powders were calcined at $900^{\circ} \mathrm{C}$ for $2 \mathrm{~h}$ and used as the raw materials to fabricate CS microspheres. The spray-drying method was deployed using a high-speed centrifugal spray-drying machine (LGZ-8; Dongsheng, Wuxi, China). We added $100 \mathrm{~g}$ CS powder into the $500 \mathrm{~g}$ aqueous solution containing $5 \mathrm{wt} \%$ polyvinyl alcohol binders. The obtained CS suspension was then atomized at a pressure of $1.5 \mathrm{MPa}$ and a flow rate of $500 \mathrm{~mL} / \mathrm{h}$. The inlet and outlet temperatures of the nozzle were adjusted to $180^{\circ} \mathrm{C}$ and $80^{\circ} \mathrm{C}$, respectively. The samples were calcined at $900^{\circ} \mathrm{C}$ for $3 \mathrm{~h}$ with a firing rate of $2^{\circ} \mathrm{C} / \mathrm{min}$ to obtain the CS microspheres. Finally, the CS microspheres were obtained by sieving the samples with a 120-150 mesh.

The nHAp microspheres were prepared by the hydrothermal treatment of the fabricated CS microspheres in the $\mathrm{Na}_{3} \mathrm{PO}_{4}$ aqueous solution using the method from our previous study. ${ }^{33}$ Then $1 \mathrm{~g}$ CS microspheres were mixed with $85 \mathrm{~mL}$ of $0.2 \mathrm{M} \mathrm{Na}_{3} \mathrm{PO}_{4}$ aqueous solution and then received a hydrothermal treatment at $180^{\circ} \mathrm{C}$ for $24 \mathrm{~h}$, as was performed in our previous study. After cooling, the samples were filtered and washed several times using deionized water. Finally, the products were obtained after being dried at $120^{\circ} \mathrm{C}$ for $24 \mathrm{~h}$.

In addition, traditional HAp microspheres with similar sizes were constructed by micro-grains as control samples. The HAp powders were first synthesized via a chemical precipitation method through the reaction of the $\left(\mathrm{NH}_{4}\right)_{2} \mathrm{HPO}_{4}$ and $\mathrm{Ca}\left(\mathrm{NO}_{3}\right)_{2} \cdot 4 \mathrm{H}_{2} \mathrm{O}$ aqueous solutions. ${ }^{33}$ The chemically precipitated HAp powders were then calcined at $900^{\circ} \mathrm{C}$ for $2 \mathrm{~h}$ and used as raw materials to fabricate the HAp microspheres via a spray-drying method.

The size and morphology of the prepared HAp microspheres as well as the prepared nHAp microspheres were measured by scanning electron microscopy (SEM). Then, the phases of the microspheres were characterized by X-ray diffraction (XRD; D/max 2,550 V; Rigaku, Tokyo, Japan) with monochromatic $\mathrm{Cu} \mathrm{K} \alpha$ radiation and Fourier transform infrared (FTIR spectra; Nicolet Co., Madison, WI, USA). The surface area of the prepared HAp and nHAp microspheres was measured on a Micromeritics Tristar 3000 system.

\section{In vitro release property of rhBMP-2}

To load the rhBMP-2 on the surfaces of the microspheres, $1 \mathrm{~g} \mathrm{nHAp}$ and HAp microspheres were immersed in $1 \mathrm{~mL}$ rhBMP-2 solution $(80 \mu \mathrm{g} / \mathrm{mL}$; Shanghai Rebone Biomaterials Co., Ltd., Shanghai, China) with acetic acid at a concentration of $0.05 \mathrm{mg} / \mathrm{mL}$ ( $80 \mu \mathrm{g}$ rhBMP-2) for $4 \mathrm{~h}$ to be totally absorbed. The rhBMP-2-loaded microspheres were freeze dried. The amount of loaded rhBMP-2 was measured by the depletion method. To investigate the release behavior of the growth factor, $0.5 \mathrm{~g}$ of the rhBMP-2-adsorbed microspheres was incubated in $2 \mathrm{~mL}$ PBS supplemented with $1 \%$ bovine serum albumin (Sigma-Aldrich Co., St Louis, MO, USA) at $37^{\circ} \mathrm{C}$ for up to 12 days with mild shaking ( $50 \mathrm{rpm}$ ). Media aliquots were periodically collected and replaced with fresh PBS. The amount of rhBMP-2 in these samples was collected and analyzed using an ELISA kit according to the manufacturer's directions (R\&D Systems, Inc., Minneapolis, MN, USA). Light absorbance was read with a microplate reader at $450 \mathrm{~nm}$.

\section{Surgical procedures}

This study adhered to the National Institutes of Health guidelines for the care and use of laboratory animals (National Institutes of Health publication no 85-23 Rev 1985) and was approved by the Research Center for Laboratory Animal of the Second Military Medical University of China. Initially, 24 male Sprague Dawley rats (National Tissue Engineering Center, Shanghai, China) weighing 400-450 g ( $\sim 14$ weeks old) were used in this study. The rats were divided into four groups with six animals in each group. Wounds were made using the method prescribed by Sánchez et al. ${ }^{34}$ In all animals, femoral bone defects, $6 \times 2 \mathrm{~mm}$, were drilled in both femora to obtain paired observations. The defect in the right leg was left empty (untreated), while the defect in the left leg (treated) was filled with different materials: pure HAp microspheres, pure nHAp microspheres, HAp microspheres with rhBMP-2, and nHAp microspheres with rhBMP-2. During the material implantation process, the weight of the implanted material was the same for each sample. In addition, during the filling operation, the material was compacted and the soft tissue on the defect surface was tightly stitched after the implantation to ensure that the material did not leak. Three rats were housed to a cage at the animal facility of the Research Center for Laboratory Animals of the Second 
Military Medical University of China. These animals were sacrificed in a $\mathrm{CO}_{2}$ chamber after implantations at 4 and 8 weeks to study their bone healing over time.

\section{Three-dimensional (3D) micro-computed tomography (CT) imaging}

An additional assay was carried out using a micro-computed tomographic imaging system (GE Healthcare Bio-Sciences Corp., Piscataway, NJ, USA). First, the femoral bone defects were scanned. Then, Microview 2.2 software was used to reconstruct the $3 \mathrm{D}$ micro- $\mathrm{CT}$ images to evaluate the bone repair process (GE Healthcare Bio-Sciences Corp.). The percentage of new bone volume (BV) relative to tissue volume (TV; BV/TV) and bone mineral density (BMD) were calculated by analyzing the bone defect site ( $\mathrm{n}=3 \mathrm{rats} / \mathrm{batch})$.

\section{Histological and histomorphometric observation}

All procedures were performed using methods found in a prior study. ${ }^{35}$ The limbs at weeks 4 and 8 were dehydrated in graded alcohol (from $75 \%$ to $100 \%$ ) and embedded in polymethyl methacrylate. An axial section of each specimen was cut and stained via van Gieson's picrofuchsin for the histological observation of new bone formation. The areas of newly formed bone were measured using a personal computer-based image analysis system (Image Pro 5.0; Media Cybernetic, Silver Springs, MD, USA) and reported as a percentage of maximum new bone area value in the experimental data.

\section{Statistical analyses}

All quantitative data were analyzed using Minitab 13 (Minitab, Inc., State College, PA, USA). The analysis of variance (ANOVA) was used for statistical comparison. Statistical significance was attained with greater than 95\% CI $(P<0.05)$.

\section{Results and discussion Characterization of fabricated $\mathrm{nHAp}$ microspheres}

Figure 1 shows the SEM images of fabricated HAp microspheres and nHAp microspheres. The low-magnification SEM images showed that the fabricated products had a uniform
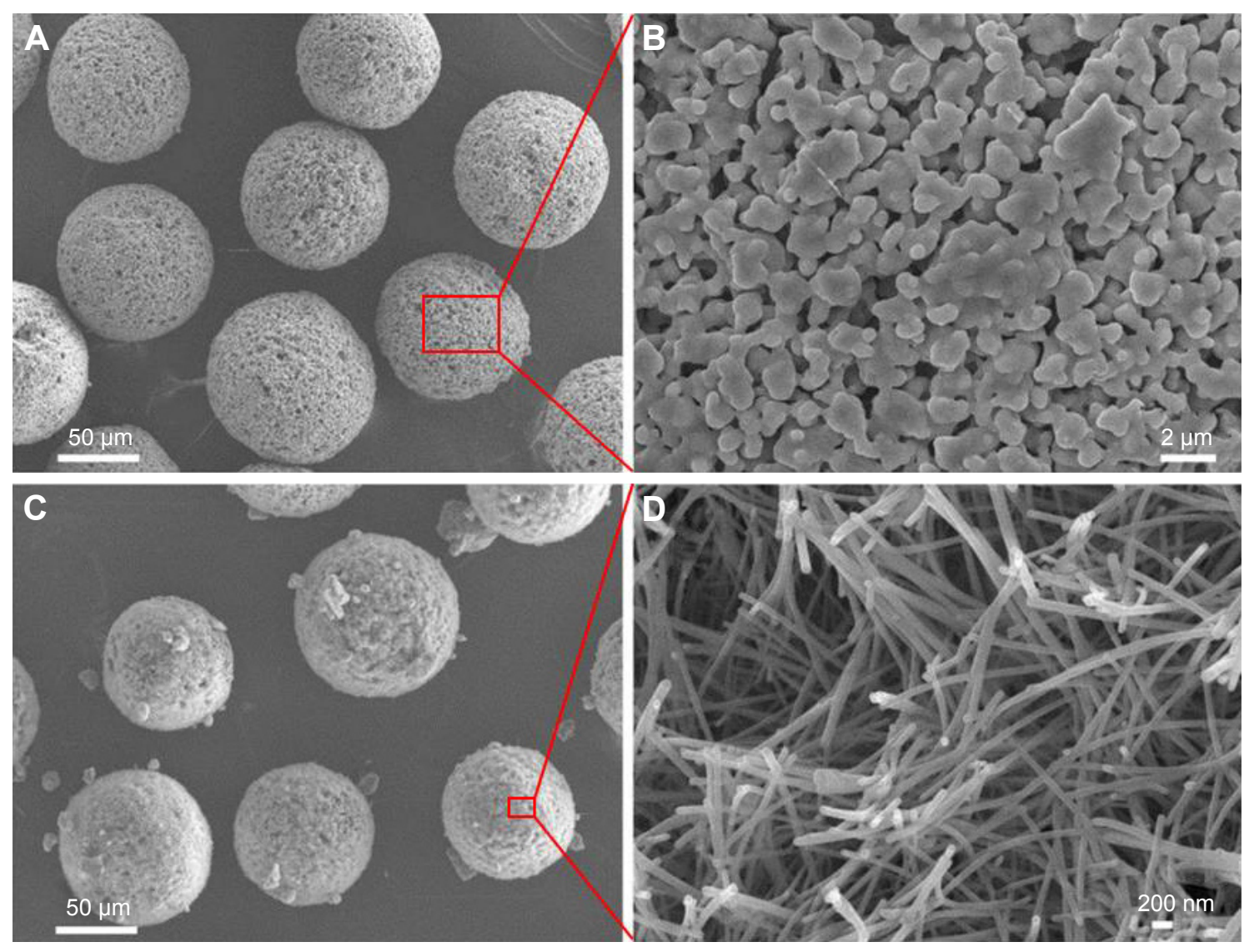

Figure I SEM morphologies of the $\mathrm{HAp}$ microspheres $(\mathbf{A}, \mathbf{B})$ and the $\mathrm{nHAp}$ microspheres obtained via hydrothermal treatment of the $\mathrm{CS}_{\text {microspheres in } \mathrm{Na}_{3} \mathrm{PO}}$ aqueous solution at $180^{\circ} \mathrm{C}$ for $24 \mathrm{~h}(\mathrm{C}, \mathrm{D})$

Abbreviations: CS, calcium silicate; HAp, hydroxyapatite; nHAp, nanostructured Hap; SEM, scanning electron microscopy. 
sphere-like morphology with a diameter of 75-100 $\mu \mathrm{m}$ (Figure 1A and C). The high-magnification SEM image showed that the HAp microspheres were composed of microparticles with a diameter of $0.3-0.6 \mu \mathrm{m}$ and micropores with a size range of $0.4-2 \mu \mathrm{m}$. In addition, the micropores were uniformly located and distributed among the HAp microspheres (Figure 1B). The nHAp microspheres hydrothermally transformed from the CS microspheres were composed of nanofibers with diameters of 80-150 nm and had lengths of several micrometers (Figure 1D). These nanofibers crossed each other to form hierarchical microspheres, and this type of structure markedly enhanced the surface-area-to-volume ratio, resulting in higher drug loading. ${ }^{36,37}$ The specific surface area: Brunauer-EmmettTeller $\left(\mathrm{S}_{\mathrm{BET}}\right)$ determination results further confirmed that nHAp microspheres transformed from the CS microspheres reached a high value of $56.14 \mathrm{~m}^{2} / \mathrm{g}$, while the HAp microspheres prepared by the traditional spray-drying method using the HAp powder as a raw material only attained a value of $3.73 \mathrm{~m}^{2} / \mathrm{g}$. Our results suggest that the novel hydrothermal method using CS microspheres as precursors provides a route to produce hierarchical nHAp with a defined matrix morphology. More importantly, the synthesized nHAp retains the original microsphere morphology, resulting in excellent biocompatibility and acting as injectable bone-filling implants. ${ }^{32,38}$

The XRD pattern showed that the CS microspheres obtained from the spray-drying method were in the pure crystalline $\beta-\mathrm{CaSiO}_{3}$ ( $\beta$-HAp) phase Joint Committee on Powder Diffraction Standards (JCPDS card No. 84-0655; Figure 2A). After the hydrothermal treatment in the $\mathrm{Na}_{3} \mathrm{PO}_{4}$ aqueous solution at $180^{\circ} \mathrm{C}$ for $24 \mathrm{~h}$, the $\beta-\mathrm{CaSiO}_{3}$ phase completely

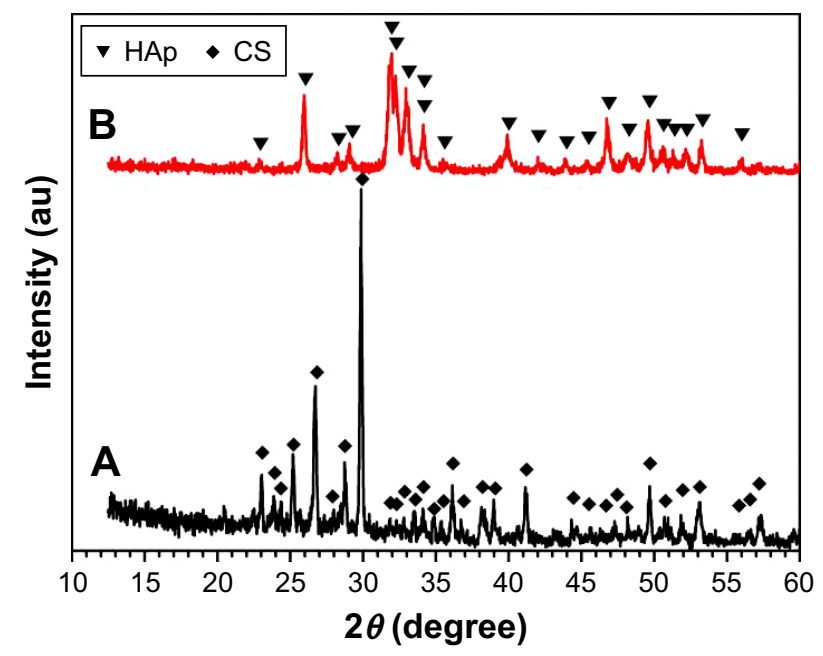

Figure 2 XRD patterns of the CS microspheres $(\mathbf{A})$ and the nHAp microspheres obtained via hydrothermal treatment of the CS microspheres in $\mathrm{Na}_{3} \mathrm{PO}_{4}$ aqueous solution at $180^{\circ} \mathrm{C}$ for $24 \mathrm{~h}(\mathrm{~B})$.

Abbreviations: CS, calcium silicate; HAp, hydroxyapatite; nHAp, nanostructured hydroxyapatite; XRD, X-ray diffraction. disappeared and transformed into the hydroxyapatite phase with the typical diffraction peaks of HAp (JCPDS card no 09-0432; Figure 2B).

FTIR spectrum was further used to evaluate the fabricated nHAp microspheres. Figure 3 shows the FTIR spectra of the nHAp. The peaks at 1,094 and $1,030 \mathrm{~cm}^{-1}$ could be assigned to the phosphate group $\left(\mathrm{PO}_{4}^{3-}\right)$ of HAp. ${ }^{39,40}$ The FTIR spectra further confirmed that the obtained products were in the hydroxyapatite phase.

\section{In vitro release behavior of rhBMP-2 from the microspheres}

We measured the loading efficiency of rhBMP-2 on the HAp and nHAp microspheres for $4 \mathrm{~h}$ at a concentration of $80 \mu \mathrm{g}$ rhBMP-2 per gram of microspheres. The results showed that more amount of the rhBMP-2 protein was absorbed on the nHAp microspheres $(54.75 \mu \mathrm{g} / \mathrm{g})$ than on the HAp microspheres $(26.08 \mu \mathrm{g} / \mathrm{g}$; Figure 4A). The cumulative in vitro release curves of the HAp and nHAp microspheres over a period of 12 days are shown in Figure 4B. The relative release rate of rhBMP-2 was higher for the HAp microspheres than that loaded on the nHAp microspheres at the beginning of the experiment. More than $90 \%$ of the protein was released within the first 2 days, and the half-life of BMP-2 release in the HAp group was about $5 \mathrm{~h}$ in vitro release study. However, nHAp released rhBMP-2 relatively slowly, even after 6 days of releasing the protein. The half-life of BMP-2 release was about $36 \mathrm{~h}$, suggesting that the nHAp microspheres can offer the sustained release of rhBMP-2.

In addition, the effective rhBMP-2 loading rate of the nHAp microspheres was significantly higher than that

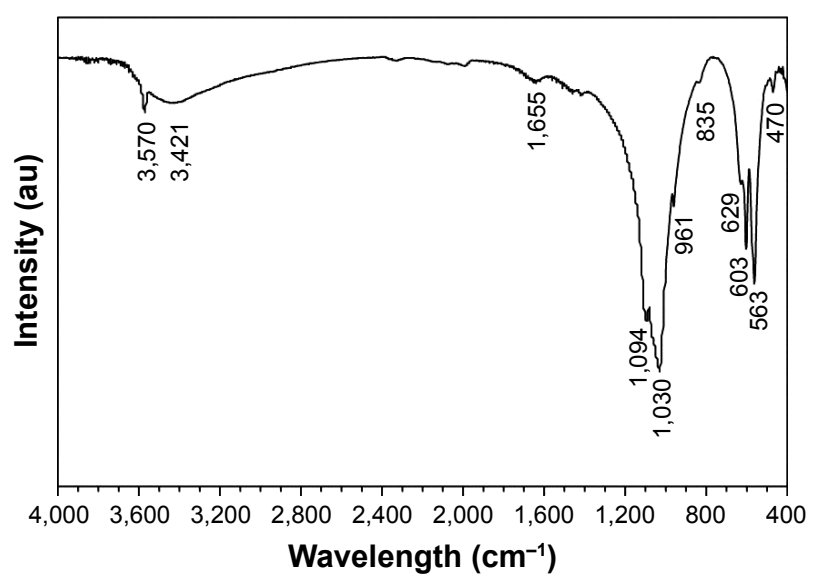

Figure 3 FTIR spectrum of the $\mathrm{nHAp}$ microspheres obtained via hydrotherma treatment of the CS microspheres in $\mathrm{Na}_{3} \mathrm{PO}_{4}$ aqueous solution at $180^{\circ} \mathrm{C}$ for $24 \mathrm{~h}$. Abbreviations: CS, calcium silicate; FTIR, Fourier transform infrared; nHAp, nanostructured hydroxyapatite. 
A

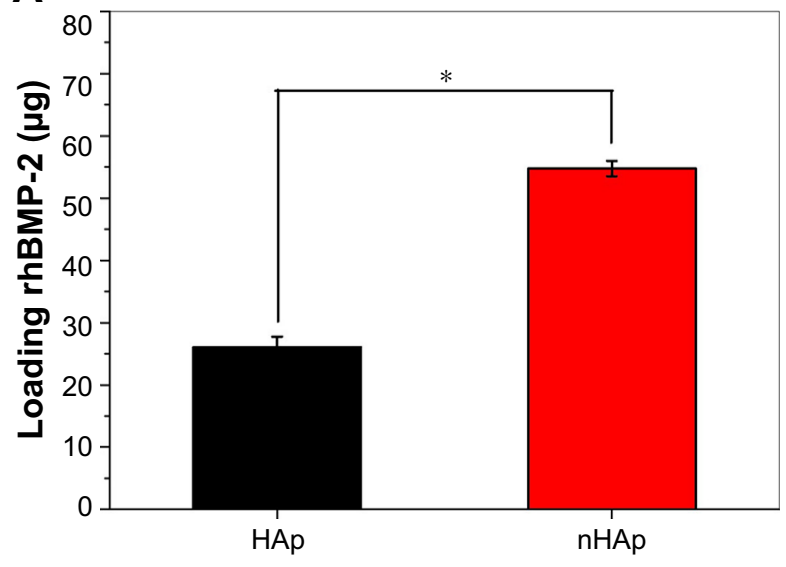

B

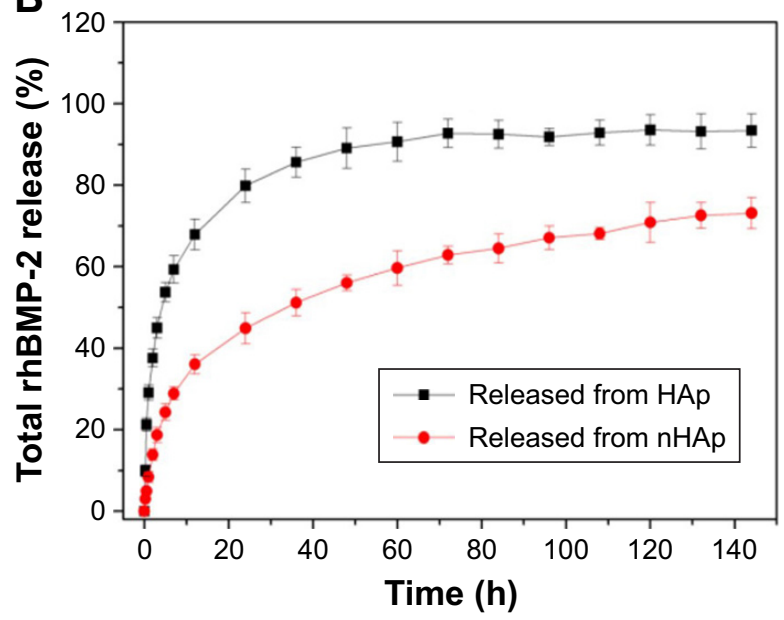

Figure 4 The amount of loaded rhBMP-2 on I g HAp and nHAp microspheres (A). Cumulative in vitro release curves of the HAp and nHAp microspheres over a period of 12 days $(\mathbf{B})$. *Significant differences, $P<0.05$.

Abbreviations: HAp, hydroxyapatite; nHAp, nanostructured HAp; rhBMP-2, recombinant human bone morphogenetic protein-2.

of the HAp microspheres. According to our analysis, the hydroxyapatite microspheres prepared via the hydrothermal conversion resulted in a nanostructured morphology that significantly improved the ability of the material to adsorb bioactive proteins. We found that the water-bridged H-bond plays an important role. It is the main force for the group interactions between the protein molecules and hydroxyapatite surface. Three types of adsorptive groups, - $\mathrm{OH},-\mathrm{NH} 2$, and -COO, are shown to faciltate BMP-2 linking to HAp surface. Among the three groups, $-\mathrm{OH}$ and $-\mathrm{NH} 2$, which have no net charge, complete their adsorption with the help of the water-bridged H-bond. ${ }^{41}$ Some studies have reported that this type of adsorption does not have a significant effect on BMP-2 activity. ${ }^{42}$ Compared to the HAp without micro/ nanostructures, the nHAp has a larger specific surface area and pore size and has more opportunities to interact with BMP-2, thereby increasing the amount of BMP-2 loading. The nanostructure offers the effective loading of the target protein, which reduces its initial burst release to prolong the duration of its residence at the site of the bone injury. This prolonged release can overcome the inherent problems associated with traditional scaffold materials. Thus, the microspheres facilitated the sustained release of the protein molecules into the medium over a relatively long time. This long duration is consistent with the results of Wang et al, ${ }^{43}$ who used an HAp nanostructure assembled on an organic porous scaffold as an absorption/release platform. This study was a new research avenue for cell growth factor release and offered a promising strategy for the design and preparation of bioactive scaffolds for bone tissue engineering.

\section{Repair of femoral supracondylar bone defects in rat models}

We further validated the ability of the HAp microspheres, nHAp microspheres, rhBMP-2-loaded HAp microspheres, and rhBMP-2-loaded nHAp microspheres to repair bone defects. A femoral bone defect $\sim 2 \times 6 \mathrm{~mm}$ in diameter was produced in the femoral intercondylar space of the rats. Micro-CT, BMD, new BV, and histological analyses were used to evaluate the repair of the bone defects with microspheres at weeks 4 and 8 after implantation.

Representative micro-CT images of 3D structures containing the repaired radii show very different orthotopic bone formations for the four groups (where the blue part is the implanted material and the yellow part is new bone; Figure 5A). In the rhBMP-2-free group, bone defects with the implantation of pure HAp and nHAp microspheres showed only slight radiopacity within 4 weeks. This result suggested only minor neo-bone formation. In the other groups with rhBMP-2, newly formed bone was observed as early as 4 weeks after implantation. The yellow area represented the new bone quantity; it was significantly higher in the rhBMP-2-loaded nHAp group than in the rhBMP-2-loaded HAp group. However, the yellow areas further increased until the bone defects were almost fully repaired after 8 weeks in the rhBMP-2-loaded nHAp group.

The radiopacity in the $3 \mathrm{D}$ micro-CT images was used to estimate the regenerated BV and BMD within the defect. The rhBMP-2-containing groups exhibited significantly greater BV than the rhBMP-2-free group (Figure 5B and C). 

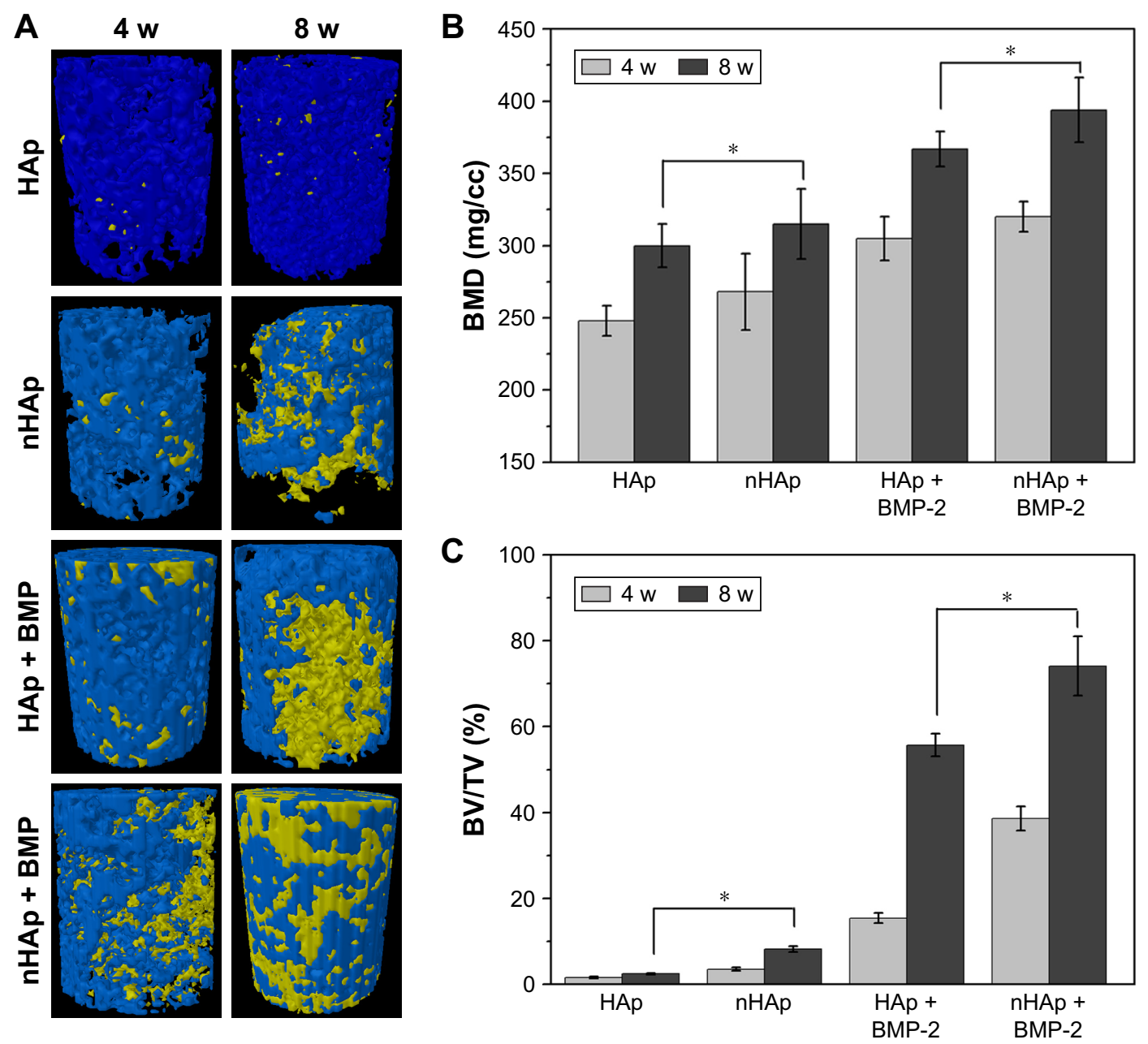

Figure 5 3D micro-CT reconstruction of bone regeneration in the femoral bone defect from animals with the most new bone formation (A). The defect sites were analyzed to calculate the BMD (B) and the percentage of new BV relative to TV (BV/TV) $(\mathbf{C})(\mathrm{n}=3$ rats/batch). *Significant differences, $P<0.05$.

Abbreviations: BMP, bone morphogenetic protein; HAp, hydroxyapatite; nHAp, nanostructured HAp; BMD, bone mineral density; BV, bone volume; CT, computed tomography; 3D, three-dimensional; TV, tissue volume; w, weeks.

In particular, the rhBMP-2-loaded nHAp group demonstrated a BV/TV value that was higher than that of the rhBMP-2loaded HAp group at both 4 and 8 weeks. There was a significant increase in the BMD value in all groups at 8 weeks. At week 8, the rhBMP-2-loaded nHAp microspheres had the highest BMD value of all groups, which confirmed the micro-CT results. This result also demonstrated that the nHAp microspheres improved the loading capacity of rhBMP-2 and maintained its sustained release. These data further confirmed the importance of the interaction between the implanted nanostructure materials and the neighboring extracellular environment. ${ }^{44}$

\section{The histomorphometric assay}

Histological analysis was based on Gieson staining and confirmed the micro-CT findings of new bone formation at 4 and 8 weeks (Figure 6A). In the rhBMP-2-free groups, the HAp group had little new bone tissue (it was only on the periphery of the bone defect sites), while much more new bone tissue was detected in the nHAp group at 4 weeks. When the implantation time reached 8 weeks, more new bone tissue was found in both the HAp and nHAp microsphere groups. Most importantly, the rhBMP-2-loaded groups possessed much more newly formed bone than those groups without rhBMP-2.

The results of the histomorphometric assay showed a higher percentage of new bone in the rhBMP-2-loaded HAp group (44\%) and rhBMP-2-loaded nHAp group (56.6\%), as compared to rhBMP-2-free HAp groups (21.2\%) and rhBMP-2-free nHAp group (24.7\%) at 4 weeks (Figure 6B). There was a significant difference between the HAp groups and the nHAp groups $(P<0.05)$. At week 8 (Figure 6C), the newly formed bone in the rhBMP-2-loaded HAp group (79.1\%) and rhBMP-2-loaded nHAp group (100\%) was 
A

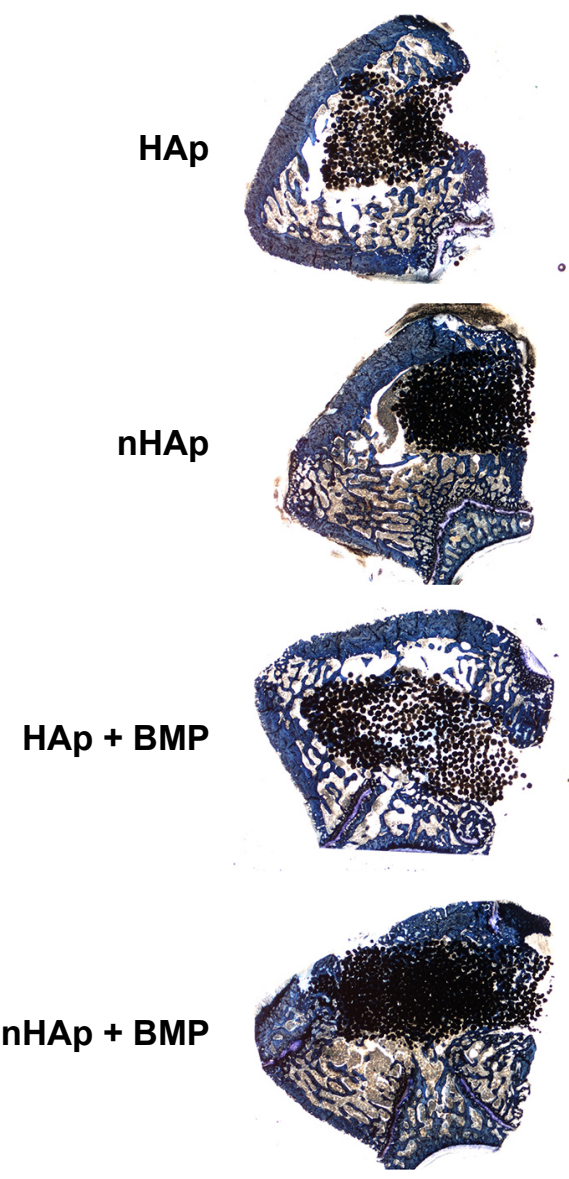

4
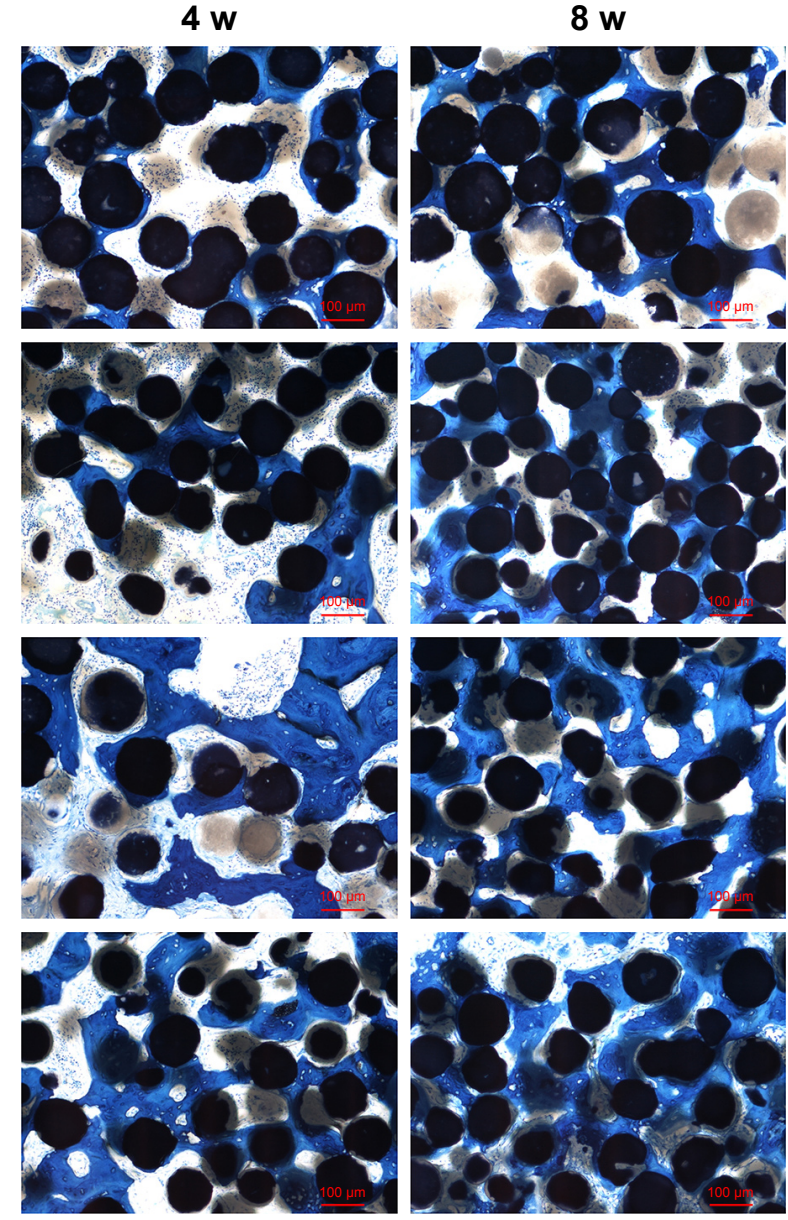

B

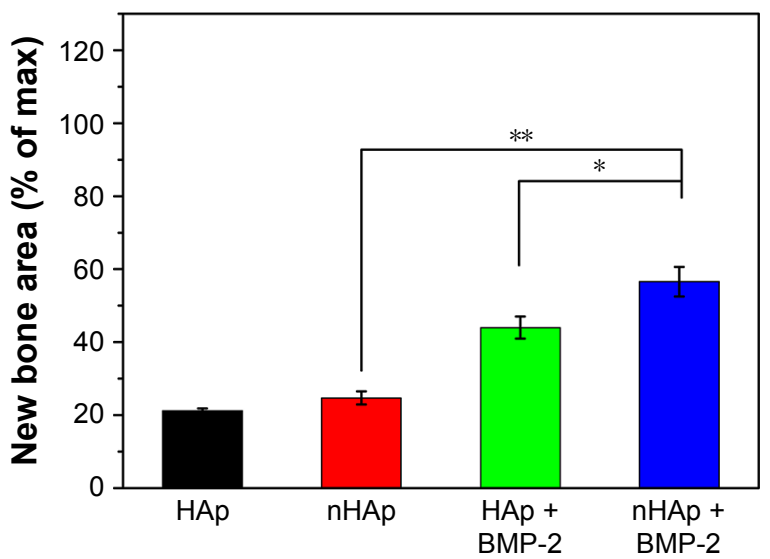

C

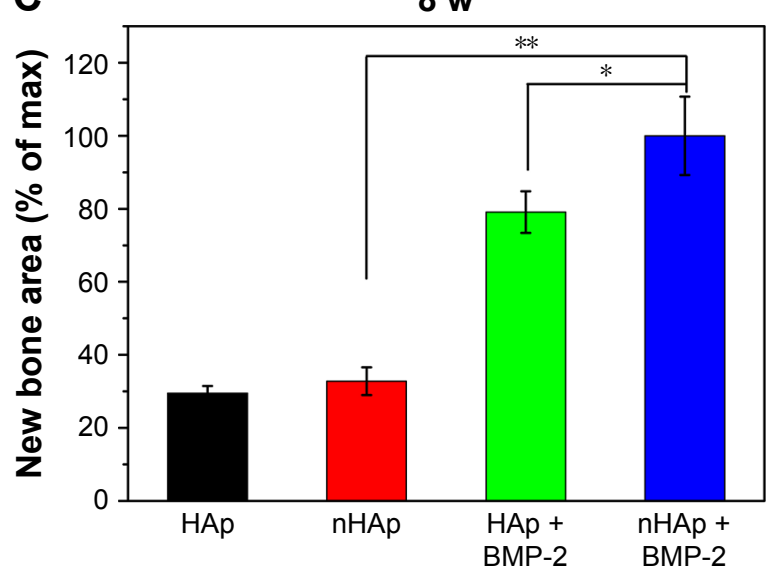

Figure 6 Histological images of newly formed bone in femoral bone defect at 4 and 8 weeks after operation (A). The percentage of new bone area assessed at 4 (B) and 8 weeks $(\mathbf{C})$ after implantation by histomorphometric analysis. All the new bone area data were normalized to the percentage of maximum new bone area value. *Significant differences, $P<0.05$; scale bar $=100 \mu \mathrm{m}$. **Significant differences, $P<0.01$.

Abbreviations: BMP-2, bone morphogenetic protein-2; HAp, hydroxyapatite; nHAp, nanostructured HAp; w, weeks.

more than that in the rhBMP-2-free HAp group (29.5\%) and rhBMP-2-free nHAp group (32.8\%). There was also a significant difference between the rhBMP-2-loaded nHAp group and rhBMP-2-loaded HAp group $(P<0.05)$. A previous study demonstrated that BMP-2 loaded on traditional HAp microspheres could significantly improve bone healing. ${ }^{45,46}$ However, the delivery and release of BMP-2 in vivo affected its application. The samples with micro/nanostructures can control the behavior of rhBMP-2, which improved its ability to induce osteoblastic differentiation. ${ }^{47}$ 
In this study, we prepared nHAp microspheres with a nanostructure that could enhance the loading ability of rhBMP-2 and prolong its release time. Our results show that the rhBMP-2-loaded nHAp microspheres containing nanostructures have a better ability to induce osteogenesis in vivo than HAp microspheres loaded with rhBMP-2. Our results indicate that the surface nanostructures formed by the hydrothermal reaction improved the osteogenic activity of the microspheres, while the surface nanostructures of the nHAp microspheres increased the loading of rhBMP-2 and delayed the release of rhBMP-2. These outcomes take full advantage of the BMP-2 activity and significantly promote the formation of new bone. Our results provide unequivocal proof that the nHAp microspheres with nanostructures can act as an excellent injectable platform for drug loading and sustained drug release, ultimately promoting bone regeneration, especially for bone defects with an irregular shape.

\section{Conclusion}

In this study, nHAp microspheres hydrothermally transformed from CS microspheres were constructed by nanofibers, which significantly improved the loading ability of rhBMP-2 and reduced its initial burst release. The prolonged duration of their residence at the site of the bone injury effectively enhanced the availability of BMP-2 in inducing osteogenesis and promoted the repair of the bone defect. The delivery and release of rhBMP-2 in vivo reduced the activity to induce osteoblastic differentiation and affected its application. The nHAp microspheres with nanostructures could effectively take full advantage of rhBMP-2 in inducing bone formation. Therefore, these nHAp microspheres have considerable advantages as carriers for bone tissue regeneration.

\section{Acknowledgments}

We acknowledge the support of the National Science Foundation for Young Scientists of China (no 81601910), the Science Foundation for Postdoctoral Research from the Secondary Military Medical University and China Postdoctoral Science Foundation Grant (no 2016M592936), the Shanghai RisingStar Program (no 18QA1405400), and the Shanghai Sailing Program (no 17YF1425000).

\section{Disclosure}

The authors report no conflicts of interest in this work.

\section{References}

1. Bohner M. Resorbable biomaterials as bone graft substitutes. Mater Today. 2010;13(1-2):24-30.
2. Marino JT, Ziran BH. Use of solid and cancellous autologous bone graft for fractures and nonunions. Orthop Clin North Am. 2010;41(1): $15-26$.

3. Sakkas A, Wilde F, Heufelder M, et al. Autogenous bone grafts in oral implantology-is it still a "gold standard"? A consecutive review of 279 patients with 456 clinical procedures. Int J Implant Dent. 2017; 3(1):23.

4. Nkenke E, Neukam FW. Autogenous bone harvesting and grafting in advanced jaw resorption: morbidity, resorption and implant survival. Eur J Oral Implantol. 2014;7(Supp1 2):S203.

5. Pape HC, Evans A, Kobbe P. Autologous bone graft: properties and techniques. J Orthop Trauma. 2010;24(Suppl 1):S36.

6. Long WGD, Einhorn TA, Koval K, et al. Bone grafts and bone graft substitutes in orthopaedic trauma surgery. J Bone Joint Surg. 2007; 89(3):649-658.

7. Xu J, Xu CR, Liu BH, Tian J. Various materials in bone grafting therapy for bone defects. J Clin Rehabil Tissue Eng Res. 2008;12(45): 8997-9000.

8. Zuo J, Kang JM, Pan L. Application of allogeneic bone transplantation in the repair of bone defects. Chin J Tissue Eng Res. 2012;16(18): 3395-3398.

9. Simion M, Fontana F. Autogenous and xenogeneic bone grafts for the bone regeneration. A literature review. Minerva Stomatol. 2004;53(5): 191-206.

10. Niederauer GG, Lee DR, Sankaran S. Bone grafting in arthroscopy and sports medicine. Sports Med Arthrosc. 2006;14(3):163-168.

11. Liu X, Ma PX. Polymeric scaffolds for bone tissue engineering. Ann Biomed Eng. 2004;32(3):477-486.

12. Muschler GF, Nakamoto C, Griffith LG. Engineering principles of clinical cell-based tissue engineering. J Bone Joint Surg Am. 2004; 86-a(7):1541-1558.

13. Zhou P, Xia Y, Cheng X, Wang P, Xie Y, Xu S. Enhanced bone tissue regeneration by antibacterial and osteoinductive silica-HACCzein composite scaffolds loaded with rhBMP-2. Biomaterials. 2014; 35(38):10033-10045.

14. Li B, Yoshii T, Hafeman AE, Nyman JS, Wenke JC, Guelcher SA. The effects of rhBMP-2 released from biodegradable polyurethane/ microsphere composite scaffolds on new bone formation in rat femora. Biomaterials. 2009;30(35):6768-6779.

15. Lazzarini L, Mader JT, Calhoun JH. Osteomyelitis in long bones. J Bone Joint Surg Am. 2004;86-a(10):2305-2318

16. Riley EH, Lane JM, Urist MR, Lyons KM, Lieberman JR. Bone morphogenetic protein-2: biology and applications. Clin Orthop Relat Res. 1996;324(324):39.

17. Ngo TQ, Scherer MA, Zhou FH, Foster BK, Xian CJ. Expression of bone morphogenic proteins and receptors at the injured growth plate cartilage in young rats. $J$ Histochem Cytochem. 2006;54(8):945-954.

18. Laflamme C, Rouabhia M. Effect of BMP-2 and BMP-7 homodimers and a mixture of BMP-2/BMP-7 homodimers on osteoblast adhesion and growth following culture on a collagen scaffold. Biomed Mater. 2008;3(1):015008.

19. Chen SH, Wang XL, Xie XH, et al. Comparative study of osteogenic potential of a composite scaffold incorporating either endogenous bone morphogenetic protein-2 or exogenous phytomolecule icaritin: an in vitro efficacy study. Acta Biomater. 2012;8:3128-3137.

20. Bessa PC, Casal M, Reis RL. Bone morphogenetic proteins in tissue engineering: the road from the laboratory to the clinic, part I (basic concepts). J Tissue Eng Regen Med. 2008;2(1):1-13.

21. Yuan HP, Fernandes H, Habibovic P, et al. Osteoinductive ceramics as a synthetic alternative to autologous bone grafting. Proc Natl Acad Sci US A. 2010;107(31):13614-13619.

22. Yuan H, Kurashina K, de Bruijn DJ, Li Y, de Groot K, Zhang X. A preliminary study of osteoinduction of two kinds of calcium phosphate bioceramics. Biomaterials. 1999;20(19):1799-1806.

23. Woodard JR, Hilldore AJ, Lan SK, et al. The mechanical properties and osteoconductivity of hydroxyapatite bone scaffolds with multi-scale porosity. Biomaterials. 2007;28(1):45-54. 
24. Liu X, Zhu L, Zhao T, et al. Synthesis and characterization of sulfonic acid-functionalized SBA-15 for adsorption of biomolecules. Microporous Mesoporous Mater. 2011;142(2-3):614-620.

25. Zhao D, Feng J, Huo Q, et al. Triblock copolymer syntheses of mesoporous silica with periodic 50 to 300 angstrom pores. Science. 1998; 279(5350):548.

26. Yang P, Zhao D, Margolese DI, et al. Generalized syntheses of largepore mesoporous metal oxides with semicrystalline frameworks. Nature. 1998;396(6707):152-155.

27. Zhou P, Cheng X, Xia Y, et al. Organic/inorganic composite membranes based on poly(1-lactic-co-glycolic acid) and mesoporous silica for effective bone tissue engineering. ACS Appl Mater Interfaces. 2014; 6(23):20895-20903

28. Lin KL, Xia LG, Gan JB, et al. Tailoring the nano-structured surfaces of hydroxyapatite bioceramics to promote protein adsorption, osteoblast growth and osteogenic differentiation. ACS Appl Mater Interfaces. 2013;5(16):8008-8017.

29. Xia L, Lin K, Jiang X, et al. Enhanced osteogenesis through nanostructured surface design of macroporous hydroxyapatite bioceramic scaffolds via activation of ERK and p38 MAPK signaling pathways. J Mater Chem B. 2013;1(40):5403-5416.

30. Xia L, Zhang N, Wang X, et al. The synergetic effect of nano-structures and silicon-substitution on the properties of hydroxyapatite scaffolds for bone regeneration. J Mater Chem B. 2016;4(19):3313-3323.

31. Dai C, Guo H, Lu J, Shi J, Wei J, Liu C. Osteogenic evaluation of calcium/magnesium-doped mesoporous silica scaffold with incorporation of rhBMP-2 by synchrotron radiation-based muCT. Biomaterials. 2011;32(33):8506-8517.

32. Lin K, Dong Z, Zhang N, Kawazoe N, Chen G, Jiang C. Fabrication and characterization of bioactive calcium silicate microspheres for drug delivery. Ceram Int. 2014;40(2):3287-3293.

33. Lin K, Chang J, Liu X, Chen L, Zhou Y. Synthesis of element-substituted hydroxyapatite with controllable morphology and chemical composition using calcium silicate as precursor. CrystEngComm. 2011;13(15): 4850-4855.

34. Sánchez E, Baro M, Soriano I, Perera A, Evora C. In vivo-in vitro study of biodegradable and osteointegrable gentamicin bone implants. Eur J Pharm Biopharm. 2001;52(2):151-158.

35. Xia L, Lin K, Jiang X, et al. Effect of nano-structured bioceramic surface on osteogenic differentiation of adipose derived stem cells. Biomaterials. 2014;35(30):8514-8527.

36. Yoo HS, Kim TG, Park TG. Surface-functionalized electrospun nanofibers for tissue engineering and drug delivery. Adv Drug Deliv Rev. 2009;61(12):1033-1042.
37. Hu X, Liu S, Zhou G, Huang Y, Xie Z, Jing X. Electrospinning of polymeric nanofibers for drug delivery applications. J Control Release. 2014; 185:12-21.

38. Xu S, Lin $\mathrm{K}$, Wang Z, et al. Reconstruction of calvarial defect of rabbits using porous calcium silicate bioactive ceramics. Biomaterials. 2008;29(17):2588-2596.

39. Lin KL, Chang J, Wang Z. Fabrication and the characterization of the bioactivity and degradability of macroporous calcium silicate bioceramics in vitro. J Inorg Mater. 2005;20(3):692-698.

40. Lin K, Zhang M, Zhai W, Qu H, Chang J. Fabrication and characterization of hydroxyapatite/wollastonite composite bioceramics with controllable properties for hard tissue repair. J Am Ceram Soc. 2011; 94(1):99-105.

41. Dong X, Wang Q, Wu T, Pan H. Understanding adsorption-desorption dynamics of BMP-2 on hydroxyapatite (001) surface. Biophys J. 2007; 93(3):750-759

42. Kang SW, Kim JS, Park KS, et al. Surface modification with fibrin/ hyaluronic acid hydrogel on solid-free form-based scaffolds followed by BMP-2 loading to enhance bone regeneration. Bone. 2011;48(2): 298-306.

43. Wang G, Qiu J, Zheng L, et al. Sustained delivery of BMP-2 enhanced osteoblastic differentiation of BMSCs based on surface hydroxyapatite nanostructure in chitosan-HAp scaffold. J Biomater Sci Polymer Ed. 2014;25(16):1813-1827.

44. Cho HJ, Perikamana SK, Lee JH, et al. Effective immobilization of BMP-2 mediated by polydopamine coating on biodegradable nanofibers for enhanced in vivo bone formation. ACS Appl Mater Interfaces. 2014;6(14):11225-11235

45. Quinlan E, Thompson EM, Matsiko A, O’Brien FJ, López-Noriega A. Long-term controlled delivery of rhBMP-2 from collagen-hydroxyapatite scaffolds for superior bone tissue regeneration. J Control Release. 2015; 207:112-119.

46. Jing Z, Huanjun Z, Kai Y, Yuan Y, Changsheng L. RhBMP-2-loaded calcium silicate/calcium phosphate cement scaffold with hierarchically porous structure for enhanced bone tissue regeneration. Biomaterials. 2013;34(37):9381-9392.

47. Huang B, Yuan Y, Ding S, et al. Nanostructured hydroxyapatite surfaces-mediated adsorption alters recognition of BMP receptor IA and bioactivity of bone morphogenetic protein-2. Acta Biomater. 2015; 27:275-285.
International Journal of Nanomedicine

\section{Publish your work in this journal}

The International Journal of Nanomedicine is an international, peerreviewed journal focusing on the application of nanotechnology in diagnostics, therapeutics, and drug delivery systems throughout the biomedical field. This journal is indexed on PubMed Central, MedLine, CAS, SciSearch ${ }^{\circledR}$, Current Contents ${ }^{\circledR} /$ Clinical Medicine,
Dovepress

Journal Citation Reports/Science Edition, EMBase, Scopus and the Elsevier Bibliographic databases. The manuscript management system is completely online and includes a very quick and fair peer-review system, which is all easy to use. Visit http://www.dovepress.com/ testimonials.php to read real quotes from published authors. 